\title{
Critical charges of simple Coulomb molecular systems: One-two electron case
}

\author{
A.V. Turbiner $*$ and H. Medel Cobaxint \\ Instituto de Ciencias Nucleares, Universidad Nacional Autónoma de México, \\ Apartado Postal 70-543, 04510 México, D.F., Mexico
}

\begin{abstract}
Let us consider some Coulomb systems of several infinitely massive centers of charge Z and onetwo electrons: $(Z, e),(2 Z, e),(3 Z, e),(4 Z, e),(2 Z, e, e),(3 Z, e, e)$. It is shown that the physical, integer charges $Z=1,2, \ldots$ do not play a distinguished role in total energy and equilibrium distance of a system giving no indication to a charge quantization.

By definition, a critical charge $Z_{c r}$ for a given Coulomb system $(n Z, e)$ or $(n Z, e, e)$ is a charge which separates a domain of the existence of bound states from a domain of unbound ones (continuum). For all above-mentioned systems critical charges $Z_{c}$ as well as equilibrium geometrical configurations are found. For all studied systems there was obtained an indication to a square-root singularity at $Z=Z_{c r}$.
\end{abstract}

*Electronic address: turbiner@nucleares.unam.mx

$\dagger$ †Electronic address: medel@nucleares.unam.mx 


\section{INTRODUCTION}

One of the basic observations of fundamental physics is the quantization of electric charges of elementary particles and nuclei. The electric charges of electron and proton have opposite signs and their values coincide, neutron has zero electric charge, any nuclear electric charge is equal to proton charge multiplied by integer number. This observation is supported experimentally, gets its justification in elementary particle theory and nuclear physics. A natural question to ask is there any indication to such a quantization in atomic-molecular physics. In classical electrostatics the stable configurations of point charges are absent (the Earnshaw's theorem), zero charge looks like as a singular point where the nature of interaction changes from repulsion to attraction. Usually, at a singular charge the whole or a part of the potential vanishes. In non-relativistic quantum electrodynamics these singular charges undoubtedly continue to exist, however, a new phenomenon occurs - there are some critical charges which separate the domain of the existence of the bound states from the domain of non-existence, although the nature of potential remains unchanged. In some cases a system gets bound at a critical charge with polynomially-decaying eigenfunctions at large distances unlike standard exponentially-decaying eigenfunctions. To the best of our knowledge this phenomenon was observed for the first time for the Helium-like system (Zee). It was named as "the level hits (kicks) continuum", or as "the zero-energy state", or as "the level on the threshold of continuum". Probably, two simplest examples where such a phenomenon occurs are the Pöschl-Teller potential and the Yukawa potential.

In this paper we consider a Coulomb system of a number of infinitely massive centers of the same charge $Z$ and one-two electrons assuming that the charge $Z$ is a real parameter. The main goal of this paper is to explore a question: are integer (physical) charges $Z$ special in some sense when the total energy is studied? Another goal is to find the domain(s) in $Z$ where the system has at least one bound state. We intend to find the critical charges $Z_{c r}$ which separate the domains of existence/non-existence of bound state. The study is made in framework of the non-relativistic quantum mechanics. 


\section{GENERALITIES}

Let us consider the Coulomb molecular system which consists of $n$ fixed charges $Z$ and $k$ electrons, $(n Z, k e)$. The Hamiltonian which describes this system is written as follows

$$
\mathcal{H}=-\frac{1}{2} \sum_{a=1}^{k} \Delta_{a}+\sum_{i<j} \frac{Z^{2}}{R_{i j}}-\sum_{i=1}^{n} \sum_{a=1}^{k} \frac{Z}{r_{i a}}+\sum_{a<b}^{k} \frac{1}{r_{a b}},
$$

in a.u., where $R_{i j}$ is the distance between charge centers $i$ and $j, r_{i a}$ is the distance from $a$ th electron to $i$ th charge center, $r_{a b}$ is the distance between electrons $a$ and $b$, and $k=1,2$. If $Z=1$, the Hamiltonian describes the system of $n$ protons and one-two electrons in the Born-Oppenheimer approximation of the zero order (the protons are considered to be infinitely-massive). There are three important particular cases.

(1) Atomic-type case, $n=1$. The Hamiltonian (1) gets a form

$$
\mathcal{H}=-\frac{1}{2} \sum_{a=1}^{k} \Delta_{a}-Z \sum_{a=1}^{k} \frac{1}{r_{a}}+\sum_{a<b}^{k} \frac{1}{r_{a b}},
$$

where $r_{a}$ is the distance between $i$ th electron and the center. At $k=1$ (one-electron case) we get a hydrogen-like ion, its spectrum is known

$$
E_{N}(Z)=-\frac{Z^{2}}{2 N^{2}}
$$

where $N$ is the principal quantum number, $N=1,2, \ldots$ Discrete spectra is infinite for any $Z>0$. Critical point is at $Z_{c r}=0$. Nature of this critical point is of quite obvious - it is a singular point of the differential equation, at $Z=0$ the potential vanishes. It is worth noting that $E_{N}(Z)$ has no singularities at finite $Z$ having the pole of the second order at $Z=\infty$. For $n>1$ it is evident from physical point of view that for small $Z$ the system is unbound but gets bound for sufficiently large $Z$. Hence, there exists some $Z=Z_{c r}$. It seems established that $Z_{c r}(n=2) \approx 0.91$ (see e.g. [1, 2] and references therein) and

$Z_{c r}(n=3) \lesssim 2.1$ (see e.g. [3]). Making a rescaling of the Hamiltonian (2), $r \rightarrow \frac{r}{Z}$ we get the Hamiltonian in the form

$$
\tilde{\mathcal{H}}=-\frac{1}{2} \sum_{a=1}^{k} \Delta_{a}-\sum_{a=1}^{k} \frac{1}{r_{a}}+\frac{1}{Z} \sum_{a<b}^{k} \frac{1}{r_{a b}},
$$

and arrive immediately at the conclusion that the energy of the bound state has the second order pole at $Z=\infty$. In general, in the domain $\left[Z_{c r}, \infty\right)$ the ground state energy $E(Z)$ is smooth monotonous function of $Z$ without any indication to a charge quantization. 
(2) One-electron, molecular-type case, $k=1$. The Hamiltonian has a form (11) without the last sum. It is evident that for small $Z$ the system is bound and one critical charge coincides with the singular point of the Hamiltonian $Z_{c r}=0$, where the potential vanishes. For large $Z$ the Coulomb repulsion of charged centers gets larger than the attraction of the electron to them and a system definitely becomes unbound. Hence, the second critical charge at finite $Z_{c r}>0$ must exist. Our goal is to find this critical charge for $n=2,3,4$.

(3) Two-electron, molecular-type case, $k=2$. The Hamiltonian (1) gets a form

$$
\mathcal{H}=-\frac{1}{2}\left(\Delta_{1}+\Delta_{2}\right)+Z^{2} \sum_{i<j}^{n} \frac{1}{R_{i j}}-Z \sum_{i=1}^{n}\left(\frac{1}{r_{i 1}}+\frac{1}{r_{i 2}}\right)+\frac{1}{r_{12}} .
$$

From physical point of view it seems evident that a system is not bound for large $Z$ as well as for $Z \leq 0$. Thus, there must exist two critical points: one has be near zero, $Z \sim 0$ and another one has to be at finite $Z$. None of them is of a type of singularity of the operator (5)). Our goal is to find this critical charge for $n=2,3$.

It is necessary to introduce a formal definition of the critical charge $Z=Z_{c r}$ for molecular system. It is natural to do it in the Born-Oppenheimer approximation of the zero order when $Z$ charges are assumed to be fixed. In the case of the existence of a bound state the potential curve $E_{\text {total }}=E_{t}(R)$ has a minimum at finite internuclear distance $R=R_{\text {eq }}$. If the bound state is stable the potential energy at infinite intercenter distance is larger at $R_{e q}$. Otherwise, the bound state can be metastable globally: the system can decay and the potential energy at infinite intercenter distance is smaller than at minimum. It implies the existence of the maximum on the potential curve at some finite $R>R_{e q}$. In the case of non-existence of a bound state the potential curve has no minimum at finite distances. Hence, at critical charge $Z=Z_{c r}$ the potential curve has a saddle point at a finite distance $R$. 


\section{ONE-ELECTRON MOLECULAR SYSTEMS}

\section{A. Two center case $(2 Z, e)$}

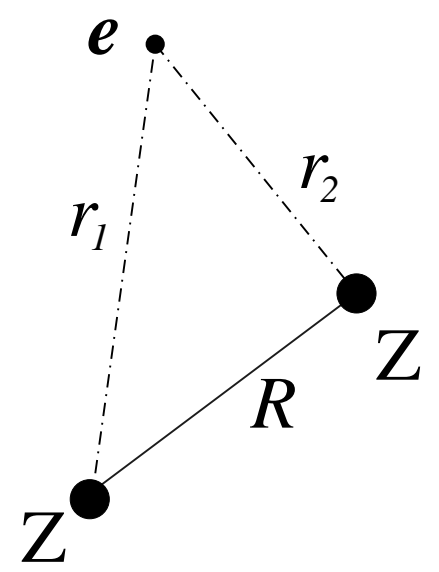

FIG. 1: Geometrical setting for $(2 Z, e)$ system

It is well known that at $Z=1$ there exists the molecular hydrogenic ion $\mathrm{H}_{2}^{+}$, while at $Z=2$ the molecular helium ion $\mathrm{He}_{2}^{3+}$ does not exist. Hence, the critical charge $Z_{c r}$ has to be in the range $1<Z<2$.

In order to calculate the total energy of $(2 Z, e)$-system vs $R$ as a function of charge $Z$ we use the variational method. As a trial function is taken linear superposition of the Heitler-London, Hund-Mulliken and Guillemin-Zener functions (see [4]).

I. The Heitler-London function.

$$
\psi_{1}=e^{-\alpha_{1} Z\left(r_{1}+r_{2}\right)}
$$

where $\alpha_{1}$ is variational parameter. It is worth mentioning the potential, for which the function $\Psi_{1}$ is exact ground state wavefunction,

$$
V_{\text {trial }}^{(1)}=-2 \alpha_{1} Z\left(\frac{1}{r_{1}}+\frac{1}{r_{2}}\right)+2 \alpha_{1}^{2} Z^{2} \overrightarrow{n_{1}} \cdot \overrightarrow{n_{2}}, E_{1}=0,
$$

reproduces both Coulomb singularities and at $\alpha_{1}=1$ even their residues. The parameter $\alpha_{1} \neq 1$ makes sense (anti)screening of the nuclear charges. It is well-known for $Z=1$ that the Heitler-London function describes small internuclear distances and can give a significant 
contribution near equilibrium, at $R \approx R_{e q}$. It mimics a coherent interaction of the electron with charged centers. It seems evident it holds for $Z \neq 1$.

II. The Hund-Mulliken function.

$$
\psi_{2}=\left(e^{-\alpha_{2} Z r_{1}}+e^{-\alpha_{2} Z r_{2}}\right)
$$

where $\alpha_{2}$ is variational parameter. It describes incoherent interaction of the electron with charged centers. This function gives a significant contribution for large internuclear distances.

In order to interpolate between domains $R \simeq R_{e q}$ and $R \gg R_{e q}$, we use two interpolating functions.

III-1. The Guillemin-Zener function

It is the simplest non-linear interpolation between $\psi_{1}$ and $\psi_{2}$ or, saying differently, between small and large internuclear distances,

$$
\psi_{3_{1}}=\left(e^{-\alpha_{3} Z r_{1}-\alpha_{4} Z r_{2}}+e^{-\alpha_{3} Z r_{2}-\alpha_{4} Z r_{1}}\right)
$$

where $\alpha_{3}, \alpha_{4}$ are variational parameters. If

- $\alpha_{3}=\alpha_{4}$ then $\psi_{3_{1}} \rightarrow \psi_{1}$

- $\alpha_{4}=0 \quad$ then $\quad \psi_{3_{1}} \rightarrow \psi_{2}$

III-2. Linear Interpolation

$$
\psi_{3_{2}}=A_{1} \psi_{1}+A_{2} \psi_{2}
$$

IV. Superposition of the two kinds of interpolation

$$
\psi_{4}=A_{3_{1}} \psi_{3_{1}}+A_{3_{2}} \psi_{3_{2}} .
$$

With such a six-parametric trial function (6) [14] the expected relative accuracy in total energy is $\approx 10^{-5}$, which is confirmed by an independent calculation based on use of highly accurate uniform approximation of the ground state eigenfunction [6] (see a discussion below). The total energy $E\left(Z, R=R_{e q}\right)$ is presented at Fig. 2 and the equilibrium distance is at Fig. 3. Both curves are smooth without any indication to a special feature at the physical charge $Z=1$. At some charge (see below) the energy curves for $(2 Z, e)$ and Z-atom 
$(Z, e)$ intersect. This crossing separates the domain of stability from metastability of the system $(2 Z, e)$. The equilibrium distance vs. $Z$ is a smooth curve which has a minimum $R_{e q}=1.952$ a.u. at $Z=0.7924$, expectedly, with a decrease of $Z$ it grows to infinity. At critical charge $Z=1.439$ the equilibrium distance, where the potential curve $E=E(R)$ has the saddle point, is equal to 2.985 a.u.

It is interesting to study the approach of the total energy to the critical charge $Z \rightarrow Z_{c r}$ from below. In order to do it we use the Puiseux expansion

$$
E_{T}(Z)=\sum_{n=0}^{\infty} a_{n}\left(Z_{c r}-Z\right)^{b_{n}}
$$

with the condition that $b_{n}<b_{n+1}$. Our goal is to find parameters $a_{n}$ and $b_{n}$ of this expansion. Restricting the expansion (17) to a finite number of terms we make fit of the total energy calculated numerically, see Table I. The fit based on data from the domain $Z \in[1.30,1.43]$ (20 points) is:

$$
\begin{aligned}
E_{T}(Z) & =-1.8072+1.5538\left(Z_{c r}-Z\right)-0.5719\left(Z_{c r}-Z\right)^{3 / 2} \\
& +0.1129\left(Z_{c r}-Z\right)^{2}+0.7777\left(Z_{c r}-Z\right)^{5 / 2}-0.4086\left(Z_{c r}-Z\right)^{5 / 2}+\ldots
\end{aligned}
$$

where the critical point is

$$
Z_{c r}^{(1)}=1.439
$$

This behavior indicates that critical point might be a square-root branch point.

There exists a charge for which a type of the binding of the system $(2 Z, e)$ is changed from metastable, $(2 Z, e) \rightarrow(Z, e)+Z$, to stable, $Z=Z_{\text {cross }}=1.237$ at $R_{\text {eq, cross }}=2.184$ a.u. It corresponds to the crossing of two potential curves on Fig. 2, If $Z<Z_{\text {cross }}$ the system $(2 Z, e)$ is stable, if $Z>Z_{\text {cross }}$ the system $(2 Z, e)$ gets unstable, $(2 Z, e) \rightarrow(Z, e)+Z$. Value of $Z_{\text {cross }}$ we calculated coincides with one found by Rebane [7].

As next we study the behavior of the total energy near the point of crossing, $Z_{\text {cross }}$. From the left , $Z<Z_{\text {cross }}$, we find as the result of the fit that the Puiseux expansion becomes the Taylor expansion

$$
E_{T}=-1.5292+1.341(1.2366-Z)+0.08(1.2366-Z)^{2}+\ldots
$$

as well as from the right, $Z>Z_{\text {cross }}$, our data are also fit by the Taylor expansion

$$
E_{T}=-1.5292+1.340(Z-1.2366)+0.05(Z-1.2366)^{2}+\ldots
$$




\begin{tabular}{|r|c|c|}
\hline$Z$ & $E_{T}$ & Fit \\
\hline \hline 0.10 & -0.031019 & -0.03071 \\
\hline 0.15 & -0.064596 & -0.06455 \\
\hline 0.20 & -0.107149 & -0.10735 \\
\hline 0.25 & -0.157038 & -0.15725 \\
\hline 0.30 & -0.212917 & -0.21287 \\
\hline 0.35 & -0.273656 & -0.27336 \\
\hline 0.40 & -0.338292 & -0.33838 \\
\hline 1.30 & -1.614220 & -1.61422 \\
\hline 1.32 & -1.641112 & -1.64111 \\
\hline 1.34 & -1.668126 & -1.66813 \\
\hline 1.36 & -1.695327 & -1.69533 \\
\hline 1.38 & -1.722801 & -1.72280 \\
\hline 1.40 & -1.750671 & -1.75067 \\
\hline 1.41 & -1.764813 & -1.76481 \\
\hline 1.42 & -1.779144 & -1.77914 \\
\hline 1.43 & -1.793737 & -1.79373 \\
\hline \hline & & \\
\hline
\end{tabular}

TABLE I: Total energy $E_{T}$ of $(2 Z, e)$ in Ry at equilibrium vs $Z$ obtained using (6) and in the method [6] compared to the result of the fit (8).

Inside of the accuracy of data used these expansions do coincide. Therefore, we do not see an indication to a branch point singularity contrary to the statement in [3]. It is worth mentioning that the dependence of $R_{e q}$ on $Z$ near $Z_{\text {cross }}$ is also very smooth, see Fig. 3 ,

Another question to rise is a behavior of the total energy near point $Z=0$ which is the singular point of the Schrödinger equation. Based on the fit of data from the domain $Z \in[0.1,0.5]$ (six points, see Table I) we find that the Puiseux expansion (7) becomes the Taylor expansion

$$
E_{T}=-3.5258 Z^{2}+4.8922 Z^{3}-3.4121 Z^{4}+\ldots
$$

Such a behavior does not provide an indication to a singular nature of the point $Z=0$. However, the total energy can not be analytically continued to $\operatorname{Re} Z<0$. 


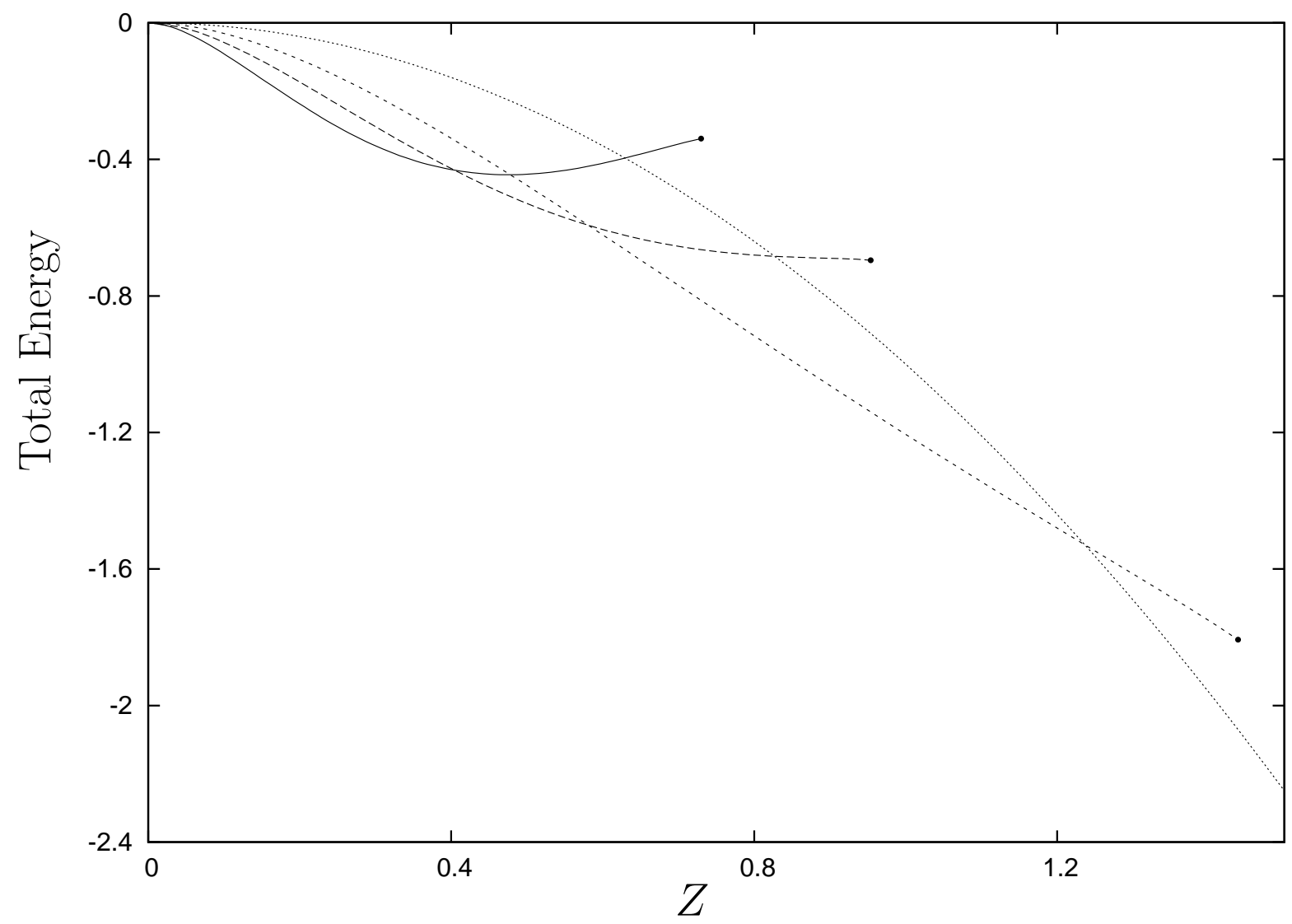

FIG. 2: Total energy in Ry of systems $(Z, e)$ (dotted line), $(2 Z, e)$ at $R=R_{e q}$ (dashed line), $(3 Z, e)$ at $R=R_{e q}$ (long-dashed line) and $(4 Z, e)$ at $R=R_{e q}$ (solid line) as functions of the charge $Z$. $(Z, e)$ and $(2 Z, e)$ curves cross at $Z=Z_{\text {cross }}=1.237$. Dashed curve ends at $Z=Z_{c r}^{(1)}=1.439$. Long-dashed curve ends at $Z=Z_{c r}^{(2)}=0.9539$. Dotted curve ends at $Z=Z_{c r}^{(3)}=0.736$

\section{B. $(3 Z, e)$}

Let us consider the electron in the electric field of three static charges $Z$ : $(3 Z, e)$. In general, these charges form triangle, see Fig. 4 as an illustration. Such a system does not exist at $Z=1$ [8]. Thus, there might exist a critical charge $Z<1$ for which the system gets bound, it separates the domain of the non-existence from existence of the bound state. Evidently, one of such critical charges is at $Z=0$, which is the singular point of the Schrödinger equation. Another one is at some $Z=Z_{c r}<1$ (see [8]). Calculations (see below) show that $Z_{c r}=0.9539$ with $R_{e q}=4.754$ a.u. Thus, the system $(3 Z, e)$ exists for charges $0<Z<Z_{c r}$ always in a form of equilateral triangle, which is the optimal geometrical configuration. It was checked that this configuration is always stable with respect to small 


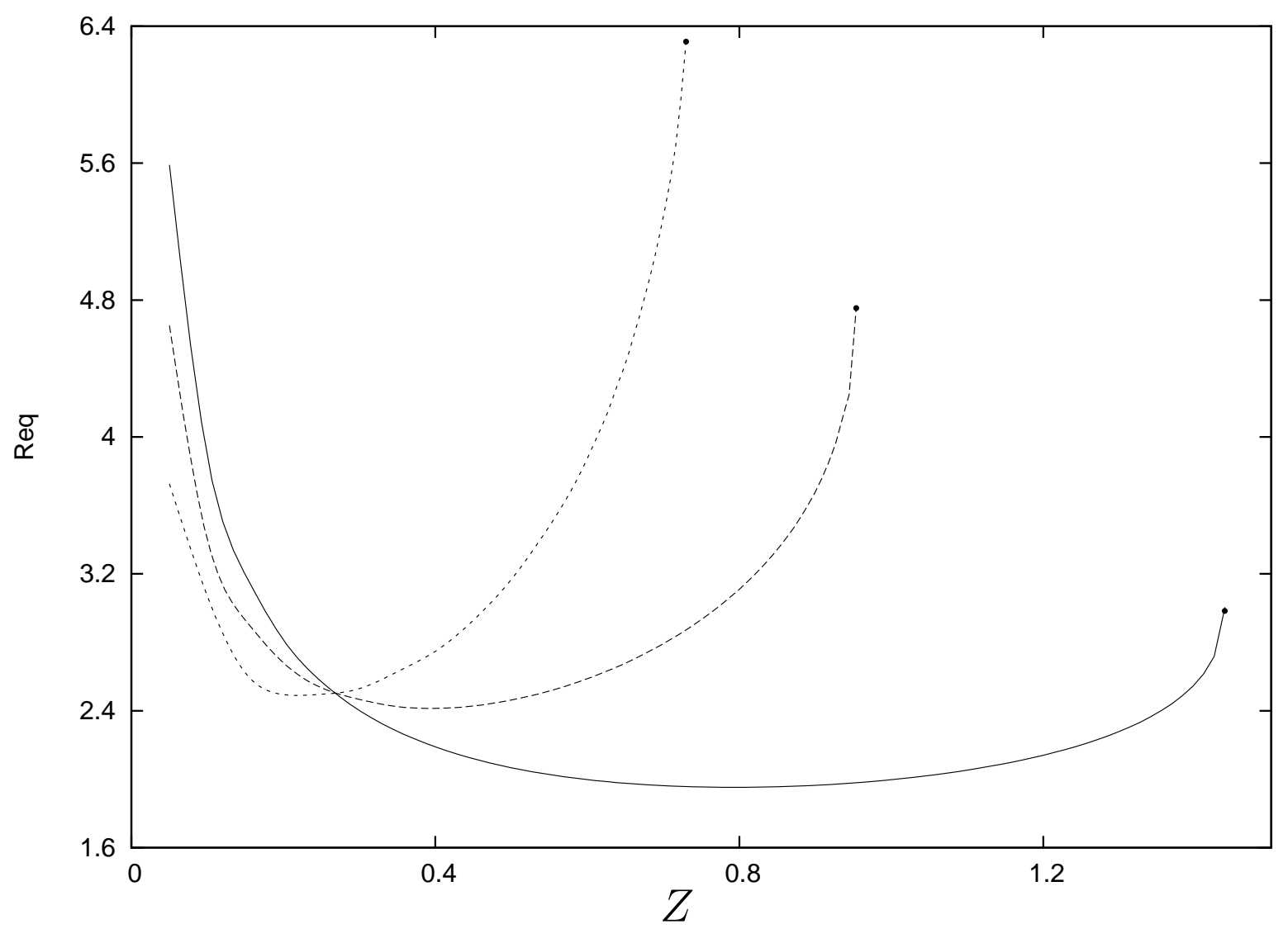

FIG. 3: Equilibrium distance in a.u. of systems $(2 Z, e)$ (dashed line), (3Z,e) (long-dashed line) and $(4 Z, e)$ (solid line) as functions of the charge $Z$. All curves cross at $Z=0.2977$ with $R_{e q}^{\text {cross }}=$ 2.366 a.u. Dashed curve ends at $Z=Z_{c r}^{(1)}=1.439$. Long-dashed curve ends at $Z=Z_{c r}^{(2)}=0.9539$. Dotted curve ends at $Z=Z_{c r}^{(3)}=0.736$.

deviation.

In order to calculate the total energy $E(Z, R)$ the variational method is used. We employ the physics-inspired trial functions [9-11] taking afterwards their linear superposition,

$$
\Psi_{\text {trial }}=\sum_{j=1}^{6} A_{j} \psi^{(j)}
$$

here $A_{j}$ are linear parameters. Each function $\psi^{(j)}$ is chosen in such a way to describe a certain physical situation of the system. In general, $\psi^{(j)}$ has the form of a symmetrized product of three $1 s$-Coulomb orbitals (Slater functions)

$$
\psi_{C}=e^{-\alpha_{1} r_{1}-\alpha_{2} r_{2}-\alpha_{3} r_{3}} .
$$

Let us give a brief description of each of them [8]: 


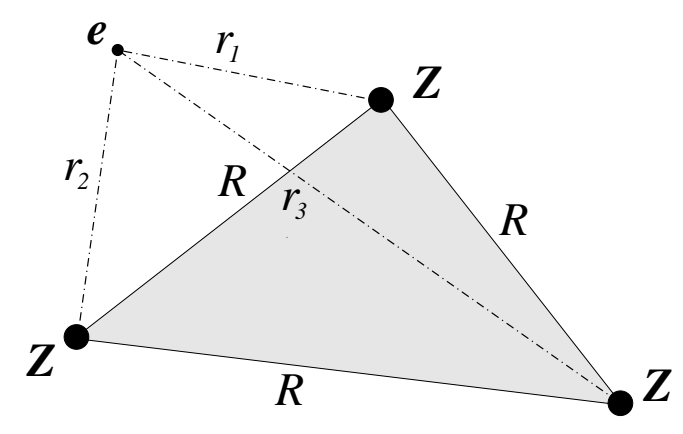

FIG. 4: Geometrical setting for $(3 Z, e)$ system

$\psi^{(1)}$ : All $\alpha$ 's are chosen to be equal to $\alpha_{1}$,

$$
\psi^{(1)}=e^{-\alpha_{1}\left(r_{1}+r_{2}+r_{3}\right)} .
$$

It is a Heitler-London type function. This corresponds to coherent interaction between the electron and all centers. Supposedly, it describes the system at small intercenter distances and, probably, the equilibrium configuration. It is verified a posteriori.

$\psi^{(2)}$ : Two $\alpha$ 's are equal to zero and the remaining one is set to be equal to $\alpha_{2}$,

$$
\psi^{(2)}=e^{-\alpha_{2} r_{1}}+e^{-\alpha_{2} r_{2}}+e^{-\alpha_{2} r_{3}} .
$$

It is a Hund-Mulliken type function. This function possibly describes the system at large distances, where essentially the electron interacts with only one center at a time thus realizing totally incoherent interaction.

$\psi^{(3)}$ : One $\alpha$ is equal to zero, two others are different from zero but equal to each other and to $\alpha_{3}$,

$$
\psi^{(3)}=e^{-\alpha_{3}\left(r_{1}+r_{2}\right)}+e^{-\alpha_{3}\left(r_{1}+r_{3}\right)}+e^{-\alpha_{3}\left(r_{2}+r_{3}\right)} .
$$

It is assumed that this function describes the system $(2 Z, e)$ plus center when a triangle is of a sufficiently small size. In fact, it is the Heitler-London function of $(2 Z, e)$-system symmetrized over centers.

$\psi^{(4)}$ : One $\alpha$ is equal to zero and two others are different from each other being equal to $\alpha_{4,5}$, respectively,

$$
\begin{aligned}
\psi^{(4)} & =e^{-\alpha_{4} r_{1}-\alpha_{5} r_{2}}+e^{-\alpha_{4} r_{2}-\alpha_{5} r_{1}}+e^{-\alpha_{4} r_{1}-\alpha_{5} r_{3}} \\
& +e^{-\alpha_{4} r_{3}-\alpha_{5} r_{1}}+e^{-\alpha_{4} r_{2}-\alpha_{5} r_{3}}+e^{-\alpha_{4} r_{3}-\alpha_{5} r_{2}} .
\end{aligned}
$$


It is assumed that this function describes the system $(2 Z, e)$ plus one center. In fact, it is the Guillemin-Zener function of the $(2 Z, e)$-system then symmetrized over centers. If $\alpha_{4}=\alpha_{5}$, the function $\psi^{(4)}$ is reduced to $\psi^{(3)}$. If $\alpha_{4}=0$, the function $\psi^{(4)}$ is reduced to $\psi^{(2)}$. Hence, $\psi^{(4)}$ is a non-linear interpolation between $\psi^{(2)}$ and $\psi^{(3)}$. It has to describe intermediate intercenter distances.

$\psi^{(5)}$ : Two $\alpha$ 's are equal but the third one is different,

$$
\begin{aligned}
\psi^{(5)} & =e^{-\alpha_{6} r_{1}-\alpha_{6} r_{2}-\alpha_{7} r_{3}}+e^{-\alpha_{6} r_{1}-\alpha_{6} r_{3}-\alpha_{7} r_{2}} \\
& +e^{-\alpha_{6} r_{2}-\alpha_{6} r_{1}-\alpha_{7} r_{3}}+e^{-\alpha_{6} r_{2}-\alpha_{6} r_{3}-\alpha_{7} r_{1}} \\
& +e^{-\alpha_{6} r_{3}-\alpha_{6} r_{1}-\alpha_{7} r_{2}}+e^{-\alpha_{6} r_{3}-\alpha_{6} r_{2}-\alpha_{7} r_{1}}
\end{aligned}
$$

It describes a "mixed" state of three Z-hydrogen atoms. If $\alpha_{6}=\alpha_{7}$, the function $\psi^{(5)}$ is reduced to $\psi^{(1)}$. If $\alpha_{6}=0$, the function $\psi^{(5)}$ is reduced to $\psi^{(2)}$. If $\alpha_{7}=0$, the function $\psi^{(5)}$ is reduced to $\psi^{(3)}$. Hence, $\psi^{(5)}$ is a non-linear interpolation between $\psi^{(1)}$, $\psi^{(2)}$ and $\psi^{(3)}$. As function (15) this is a type of Guillemin-Zener function and should describe intermediate intercenter distances.

$\psi^{(6)}$ : All $\alpha$ 's are different,

$$
\begin{aligned}
\psi^{(6)} & =e^{-\alpha_{8} r_{1}-\alpha_{9} r_{2}-\alpha_{10} r_{3}}+e^{-\alpha_{8} r_{1}-\alpha_{9} r_{3}-\alpha_{10} r_{2}} \\
& +e^{-\alpha_{8} r_{2}-\alpha_{9} r_{1}-\alpha_{10} r_{3}}+e^{-\alpha_{8} r_{2}-\alpha_{9} r_{3}-\alpha_{10} r_{1}} \\
& +e^{-\alpha_{8} r_{3}-\alpha_{9} r_{1}-\alpha_{10} r_{2}}+e^{-\alpha_{8} r_{3}-\alpha_{9} r_{2}-\alpha_{10} r_{1}} .
\end{aligned}
$$

This is a general non-linear interpolation of all functions $\psi^{(1-5)}$.

The total number of parameters of the function (10) is equal to 15, where five are linear ones. Note that without a loss of generality the parameter $A_{6}$ in (10) can be fixed, putting $A_{6}=1$. We expect this function provides a relative accuracy $\sim 10^{-3}$ in total energy.

As a result of variational study for fixed $Z$ the optimal geometric configuration is always the equilateral triangle. It was checked that this configuration is always stable with respect to small deviation. On Fig. 2 the total energy dependence for $(3 Z, e)$ at the equilibrium configuration is given. It is a smooth monotonous curve which ends at $Z=Z_{c r}<1$. At some charges this curve intersects with the energy curves for $(2 Z, e)$ and Z-atom, $(Z, e)$. These 
crossings separate domains of stability from different domains of metastability of the system (see below). On Fig. 3 the equilibrium distance between nearest static charges (the side of the equilateral triangle) is shown. It is a smooth curve which has a minimum $R_{e q}^{\min }=2.413$ a.u. at $Z=0.391$ and it grows to infinity with a decrease of $Z$. At critical charge $Z=0.9539$ the equilibrium distance, where the potential curve $E=E(R)$ has the saddle point, is equal to 4.754 a.u. It is worth mentioning that the $R_{e q}$ curves for $(3 Z, e)$ and $(2 Z, e)$ intersect at $Z=0.2670$ with $R_{e q}=2.506$ a.u.

It is interesting to study the approach of the total energy to the critical charge $Z \rightarrow Z_{c r}$ from below. In order to do it we use a general Puiseux expansion (7). Eventually, the behavior of the total energy close to critical charge $Z_{c r}$, as a result of the fit, is given by the following terminated Puiseux expansion:

$$
\begin{aligned}
E(Z)= & -0.6954+0.2700\left(Z_{c r}-Z\right)-1.0357\left(Z_{c r}-Z\right)^{3 / 2}-1.3360\left(Z_{c r}-Z\right)^{2} \\
& -0.1350\left(Z_{c r}-Z\right)^{5 / 2}+2.3395\left(Z_{c r}-Z\right)^{3}-1.8714\left(Z_{c r}-Z\right)^{7 / 2}
\end{aligned}
$$

where the critical point is

$$
Z_{c r}^{(2)}=0.9539
$$

The fit (18) is based on data from the domain $Z \in[0.80,0.93]$ (19 points, see Table II). This behavior indicates that the critical point might be a square-root branch point.

There are two points of crossing for the energy curve $(3 Z, e)$ at Fig. 2, The first one is $Z_{\text {cross }}^{(1)}=0.8269$ with $R_{e q}=3.234$ a.u. for the crossing of $(3 Z, e)$ and the $(Z, e)$. The second one is $Z_{\text {cross }}^{(2)}=0.5811$ with $R_{e q}=2.640$ a.u. for the crossing of $(3 Z, e)$ and the $(2 Z, e)$ at $R_{e q}=2.008$ a.u. For charges $Z \in(0.8269,0.9537)$ for the triangular equilateral configuration the system is metastable with two decay channels

$$
\begin{array}{r}
(3 Z, e) \rightarrow(Z, e)+Z+Z, \\
(3 Z, e) \rightarrow Z_{2}^{+}+Z,
\end{array}
$$

while for $Z \in(0.5811,0.8269)$ system is metastable with single decay channel

$$
(3 Z, e) \rightarrow(2 Z, e)+Z
$$

and, finally, for $Z<0.5811$ the system is stable. A study of the Puiseux expansions near $Z_{\text {cross }}^{(1)}$ as well as $Z_{\text {cross }}^{(2)}$ from above and below show that they are the Taylor expansions which 


\begin{tabular}{|c|c|c|}
\hline$Z$ & $E_{T}$ & Fit \\
\hline \hline 0.10 & -0.057230 & -0.056880 \\
\hline 0.15 & -0.111367 & -0.111714 \\
\hline 0.20 & -0.173248 & -0.173581 \\
\hline 0.25 & -0.238656 & -0.238205 \\
\hline 0.30 & -0.304100 & -0.304235 \\
\hline \hline 0.80 & -0.680137 & -0.680137 \\
\hline 0.82 & -0.682953 & -0.682953 \\
\hline 0.84 & -0.685194 & -0.685194 \\
\hline 0.86 & -0.686969 & -0.686969 \\
\hline 0.88 & -0.688417 & -0.688417 \\
\hline 0.90 & -0.689716 & -0.689716 \\
\hline 0.91 & -0.690385 & -0.690385 \\
\hline 0.92 & -0.691123 & -0.691123 \\
\hline 0.93 & -0.691991 & -0.691991 \\
\hline
\end{tabular}

TABLE II: Total energy $E_{T}$ of $(3 Z, e)$ in Ry at equilibrium vs $Z$ obtained using (10) compared to the result of the fit (18).

do coincide within the accuracy of data used and the obtained parameters of the fit. They do not give an indication that these points are branch points. It also is worth mentioning that the dependence of $R_{e q}$ on $Z$ near $Z_{\text {cross }}^{(1,2)}$ is also very smooth, see Fig. 3 .

Another question to rise is a behavior of the total energy near the critical point at $Z=0$ which is the singular point of the Schrödinger equation. Based on the fit of data from the domain $Z \in[0.1,0.7]$ (seven points, see Table II) we find that the Puiseux expansion becomes the Taylor expansion

$$
E_{T}=-7.4257 Z^{2}+19.3244 Z^{3}-19.4662 Z^{4}+\ldots .
$$

Such a behavior does not provide an indication to singular nature of the point $Z=0$. However, the total energy can not be analytically continued to $\operatorname{Re} Z<0$. 


\section{C. $(4 Z, e)$}

This Coulomb system consists of four static $Z$-charges and one electron, $(4 Z, e)$. It is worth anticipating that the most symmetrical configuration where $Z$-charges are placed on the vertexes of a tetrahedron, see Fig. 5, is optimal. It was checked that this configuration is always stable with respect to small deviations. It is certain that for $Z=1$ there is a bound state, the system $\mathrm{H}_{4}^{3+}$ does not exist.

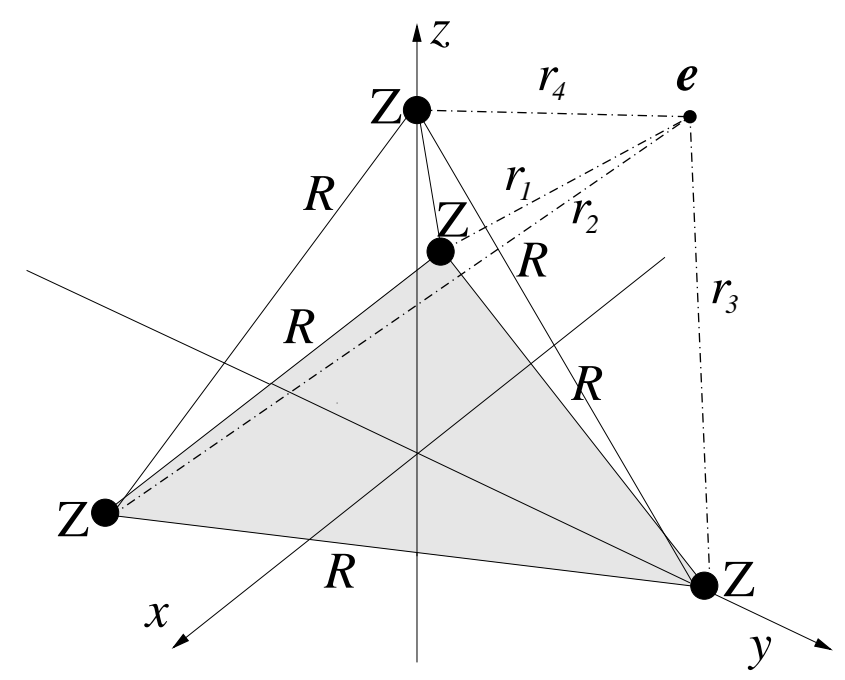

FIG. 5: Geometrical setting for $(4 Z, e)$ system

Trial Functions. The variational method was used to obtain all numerical results. Trial function is taken in a form of linear superposition of three functions:

$$
\Psi_{\text {trial }}=\sum_{j=1}^{3} A_{j} \psi^{(j)}
$$

where $A_{j}$ are linear parameters. Each function $\psi^{(j)}$ is chosen in such a way as to describe different physical characteristics of the system [9, 10]. In general, $\psi^{(j)}$ has the form of a symmetrized product of four $1 s$-Coulomb orbitals (Slater functions)

$$
\psi_{C}=e^{-\alpha_{1} r_{1}-\alpha_{2} r_{2}-\alpha_{3} r_{3}-\alpha_{4} r_{4}}
$$

Let us give a brief description of each of them:

$\psi^{(1)}:$ All $\alpha$ 's are chosen to equal to $\alpha_{1}$,

$$
\psi^{(1)}=e^{-\alpha_{1}\left(r_{1}+r_{2}+r_{3}+r_{4}\right)} .
$$


It is a Heitler-London type function. This corresponds to coherent interaction between the electron and all protons. Supposedly, it describes the system at small interproton distances and, probably, the equilibrium configuration.

$\psi^{(2)}$ : Three $\alpha$ 's are equal to zero and the remaining one is set to be equal to $\alpha_{2}$,

$$
\psi^{(2)}=e^{-\alpha_{2} r_{1}}+e^{-\alpha_{2} r_{2}}+e^{-\alpha_{2} r_{3}}+e^{-\alpha_{2} r_{4}} .
$$

It is a Hund-Mulliken type function. This function possibly describes the system at large distances, where essentially the electron interacts with only one proton at a time thus realizing incoherent interaction.

$\psi^{(3)}:$ All $\alpha^{\prime}$ s are different from each other, and different from zero.

$$
\begin{aligned}
\psi^{(3)} & =e^{-\alpha_{3} r_{1}-\alpha_{4} r_{2}-\alpha_{5} r_{3}-\alpha_{6} r_{4}}+e^{-\alpha_{3} r_{1}-\alpha_{4} r_{2}-\alpha_{6} r_{3}-\alpha_{5} r_{4}} \\
& +e^{-\alpha_{3} r_{1}-\alpha_{5} r_{2}-\alpha_{4} r_{3}-\alpha_{6} r_{4}}+e^{-\alpha_{3} r_{1}-\alpha_{5} r_{2}-\alpha_{6} r_{3}-\alpha_{4} r_{4}} \\
& +e^{-\alpha_{3} r_{1}-\alpha_{6} r_{2}-\alpha_{4} r_{3}-\alpha_{5} r_{4}}+e^{-\alpha_{3} r_{1}-\alpha_{6} r_{2}-\alpha_{5} r_{3}-\alpha_{4} r_{4}} \\
& +e^{-\alpha_{4} r_{1}-\alpha_{3} r_{2}-\alpha_{5} r_{3}-\alpha_{6} r_{4}}+e^{-\alpha_{4} r_{1}-\alpha_{3} r_{2}-\alpha_{6} r_{3}-\alpha_{5} r_{4}} \\
& +e^{-\alpha_{4} r_{1}-\alpha_{5} r_{2}-\alpha_{3} r_{3}-\alpha_{6} r_{4}}+e^{-\alpha_{4} r_{1}-\alpha_{5} r_{2}-\alpha_{6} r_{3}-\alpha_{3} r_{4}} \\
& +e^{-\alpha_{4} r_{1}-\alpha_{6} r_{2}-\alpha_{3} r_{3}-\alpha_{5} r_{4}}+e^{-\alpha_{4} r_{1}-\alpha_{6} r_{2}-\alpha_{5} r_{3}-\alpha_{3} r_{4}} \\
& +e^{-\alpha_{5} r_{1}-\alpha_{3} r_{2}-\alpha_{4} r_{3}-\alpha_{6} r_{4}}+e^{-\alpha_{5} r_{1}-\alpha_{3} r_{2}-\alpha_{6} r_{3}-\alpha_{4} r_{4}} \\
& +e^{-\alpha_{5} r_{1}-\alpha_{4} r_{2}-\alpha_{3} r_{3}-\alpha_{6} r_{4}}+e^{-\alpha_{5} r_{1}-\alpha_{4} r_{2}-\alpha_{6} r_{3}-\alpha_{3} r_{4}} \\
& +e^{-\alpha_{5} r_{1}-\alpha_{6} r_{2}-\alpha_{3} r_{3}-\alpha_{4} r_{4}}+e^{-\alpha_{5} r_{1}-\alpha_{6} r_{2}-\alpha_{4} r_{3}-\alpha_{3} r_{4}} \\
& +e^{-\alpha_{6} r_{1}-\alpha_{3} r_{2}-\alpha_{4} r_{3}-\alpha_{5} r_{4}}+e^{-\alpha_{6} r_{1}-\alpha_{3} r_{2}-\alpha_{5} r_{3}-\alpha_{4} r_{4}} \\
& +e^{-\alpha_{6} r_{1}-\alpha_{4} r_{2}-\alpha_{3} r_{3}-\alpha_{5} r_{4}}+e^{-\alpha_{6} r_{1}-\alpha_{4} r_{2}-\alpha_{5} r_{3}-\alpha_{3} r_{4}} \\
& +e^{-\alpha_{6} r_{1}-\alpha_{5} r_{2}-\alpha_{3} r_{3}-\alpha_{4} r_{4}}+e^{-\alpha_{6} r_{1}-\alpha_{5} r_{2}-\alpha_{4} r_{3}-\alpha_{3} r_{4}}
\end{aligned}
$$

We can see that trial functions $\psi^{(1)}$ and $\psi^{(2)}$ are particular cases of the general trial function $\psi^{(3)}$.

There might exist two critical charges which separates the domain of existence from nonexistence of bound states. One such a critical charge is at $Z=0$. Another one is at some $Z=Z_{c r}<1$. Calculations (see below) show that $Z_{c r}=0.736$ at $R_{e q}=6.50$ a.u. , where the potential curve $E=E(R)$ has the saddle point. Thus, the system $(4 Z, e)$ can exist 
for charges $0<Z<Z_{c r}$. The energy dependence at equilibrium distance $R_{e q}$ is a smooth function, see Fig. 2. The optimal geometric configuration is always the tetrahedron. It was checked that this configuration is always stable with respect to small deviations. It is a smooth monotonous curve which ends at $Z=Z_{c r}<1$. At some charges it intersects the energy curves for $(3 Z, e),(2 Z, e)$ and Z-atom $(Z, e)$. These crossings separate domains of stability from metastability of the system (see below). On Fig. 3 the equilibrium distance between nearest static charges (the side of the tetrahedron) is shown. It is a smooth curve which has a minimum $R_{e q}=2.485$ a.u. at $Z=0.2218$ and it grows to infinity with a decrease of $Z$. It is quite amusing that all three equilibrium distance curves for $(4 Z, e),(3 Z, e)$ and $(2 Z, e)$ intersect for $Z=0.2670$ with $R_{e q}=2.506$ a.u.

Behavior of the energy as function of the charge close to critical charge $Z_{c r}$ is given by the terminated Puiseux expansion:

$$
\begin{aligned}
E(Z) & =-0.3368-0.2793\left(Z_{c r}-Z\right)-1.5995\left(Z_{c r}-Z\right)^{3 / 2} \\
& +2.0214\left(Z_{c r}-Z\right)^{2}+0.9224\left(Z_{c r}-Z\right)^{5 / 2}+\ldots
\end{aligned}
$$

where the critical point is

$$
Z_{c r}^{(3)}=0.736
$$

The fit (25) is based on data from the domain $Z \in[0.60,0.72]$ (12 points, see Table III). This behavior indicates that critical point might be a square-root branch point.

There are three points of crossing for the energy curve $(4 Z, e)$ at Fig. 2. The first one is for the crossing of $(4 Z, e)$ and the $(Z, e)$ energy curve at $Z_{\text {cross }}^{(1)}=0.6290$ with $R_{e q}=4.187$ a.u. The second one is at $Z_{\text {cross }}^{(2)}=0.4798$ with $R_{e q}=3.08$ a.u. for the crossing of $(4 Z, e)$ and the $(2 Z, e)$ energy curve at $R_{e q}=2.086$ a.u. The third one is $Z_{\text {cross }}^{(3)}=0.4065$ with $R_{e q}=2.83$ a.u. for the crossing of $(4 Z, e)$ and the $(3 Z, e)$ energy curve at $R_{e q}=2.413$ a.u.

For charges $Z \in(0.6290,0.7360)$ for the triangular equilateral configuration the system is metastable with three decay channels

$$
\begin{array}{r}
(4 Z, e) \rightarrow(Z, e)+Z+Z+Z \\
(4 Z, e) \rightarrow(2 Z, e)+Z+Z \\
(4 Z, e) \rightarrow(3 Z, e)+Z
\end{array}
$$




\begin{tabular}{|c|c|c|}
\hline$Z$ & $E_{T}$ & Fit \\
\hline \hline 0.10 & -0.092030 & -0.092063 \\
\hline 0.11 & -0.106530 & -0.106491 \\
\hline 0.12 & -0.121220 & -0.121190 \\
\hline 0.13 & -0.136020 & -0.136072 \\
\hline 0.14 & -0.151080 & -0.151070 \\
\hline 0.15 & -0.166150 & -0.166145 \\
\hline \hline 0.60 & -0.411356 & -0.411387 \\
\hline 0.62 & -0.401055 & -0.401025 \\
\hline 0.64 & -0.389997 & -0.389987 \\
\hline 0.66 & -0.378426 & -0.378456 \\
\hline 0.68 & -0.366652 & -0.366675 \\
\hline 0.70 & -0.355022 & -0.354995 \\
\hline 0.72 & -0.344089 & -0.344021 \\
\hline
\end{tabular}

TABLE III: Total energy $E_{T}$ of $(4 Z, e)$ in Ry at equilibrium vs $Z$ obtained variationally using the trial function (20) compared to the result of the fit (25).

For $Z \in(0.4798,0.6290)$ the system is metastable with two decay channels

$$
\begin{array}{r}
(4 Z, e) \rightarrow(2 Z, e)+Z+Z \\
(4 Z, e) \rightarrow(3 Z, e)+Z
\end{array}
$$

For $Z \in(0.4065,0.4798)$ system is metastable with one decay channel

$$
(4 Z, e) \rightarrow(3 Z, e)+Z
$$

and, finally, for $0<Z<0.4065$ the system gets stable.

A question to rise is about behavior of the total energy near the critical point at $Z=0$ which is the singular point of the Schrödinger equation. Based on the fit of data from the domain $Z \in[0 ., 0.15]$ (seven points, see Table III) we find that the Puiseux expansion becomes the Taylor expansion

$$
E_{T}=-14.3871 Z^{2}+62.0529 Z^{3}-102.4490 Z^{4}+\ldots .
$$


Such a behavior does not provide an indication to singular nature of the point $Z=0$. However, the total energy can not be analytically continued to $\operatorname{Re} Z<0$.

\section{TWO-ELECTRON MOLECULAR SYSTEMS}

\section{A. $(2 Z, e, e)$}

The system $(2 Z, e, e)$ consists of two charged centers $Z$ and two electrons. For $Z=1$ it is the celebrated $\mathrm{H}_{2}$ molecule when for $Z=2$ it is the Helium molecular ion $\mathrm{He}_{2}^{(++)}$which is metastable system. It is obvious that for large $Z$ the system is unbound as well as for negative $Z$. Thus, there are two singular points: $Z=0$ where the potential "changes" sign and $Z_{c r}>2$ which is a critical point separating the domain of the existence from the domain of non-existence of the solutions in the Hilbert space. It seems natural that the ground state when exists is the spin-singlet state. Calculations (see below) show that the critical charge $Z_{c r}=2.250$ at $R_{e q}=1.532$ a.u. Thus, the system $(2 Z, e, e)$ exists for charge $0<Z<Z_{c r}$.

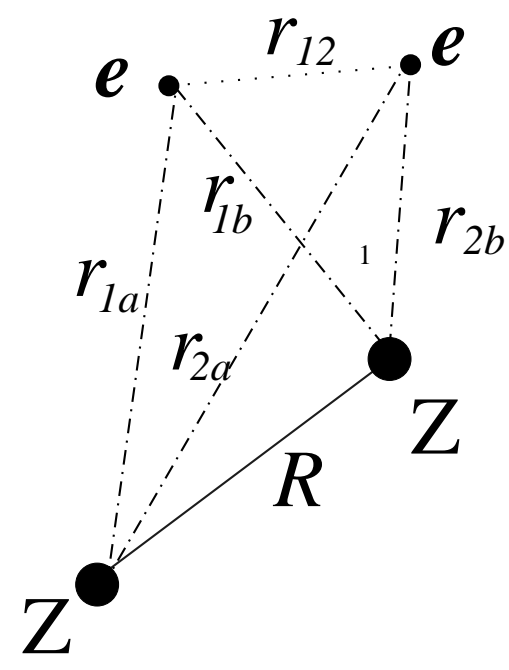

FIG. 6: Geometrical setting for $(2 Z, e, e)$ system

Trial Functions. To calculate the total energy of the $(2 Z, e, e)$ system as a function of charge $Z$ the variational method is used. Exponential correlated trial functions with proper symmetrization are employed as well as their linear superposition. In general, the basic trial function $\psi^{(j)}$ is taken in the form of symmetrized product of four $1 s$-Coulomb orbitals 
(Slater functions) and correlation function in exponential form [12],

$$
\begin{aligned}
\psi_{g} & =e^{-\alpha_{1} r_{1 a}-\alpha_{2} r_{1 b}-\alpha_{3} r_{2 a}-\alpha_{4} r_{2 b}+\gamma r_{12}} \\
& +e^{-\alpha_{3} r_{1 a}-\alpha_{4} r_{1 b}-\alpha_{1} r_{2 a}-\alpha_{2} r_{2 b}+\gamma r_{12}} \\
& +e^{-\alpha_{2} r_{1 a}-\alpha_{1} r_{1 b}-\alpha_{4} r_{2 a}-\alpha_{3} r_{2 b}+\gamma r_{12}} \\
& +e^{-\alpha_{2} r_{1 a}-\alpha_{1} r_{1 b}-\alpha_{4} r_{2 a}-\alpha_{3} r_{2 b}+\gamma r_{12}}
\end{aligned}
$$

Recently, it was shown that a linear superposition of three functions (30) leads to the most accurate ground state energy for the $\mathrm{H}_{2}$-molecule among a few parametric trial functions.

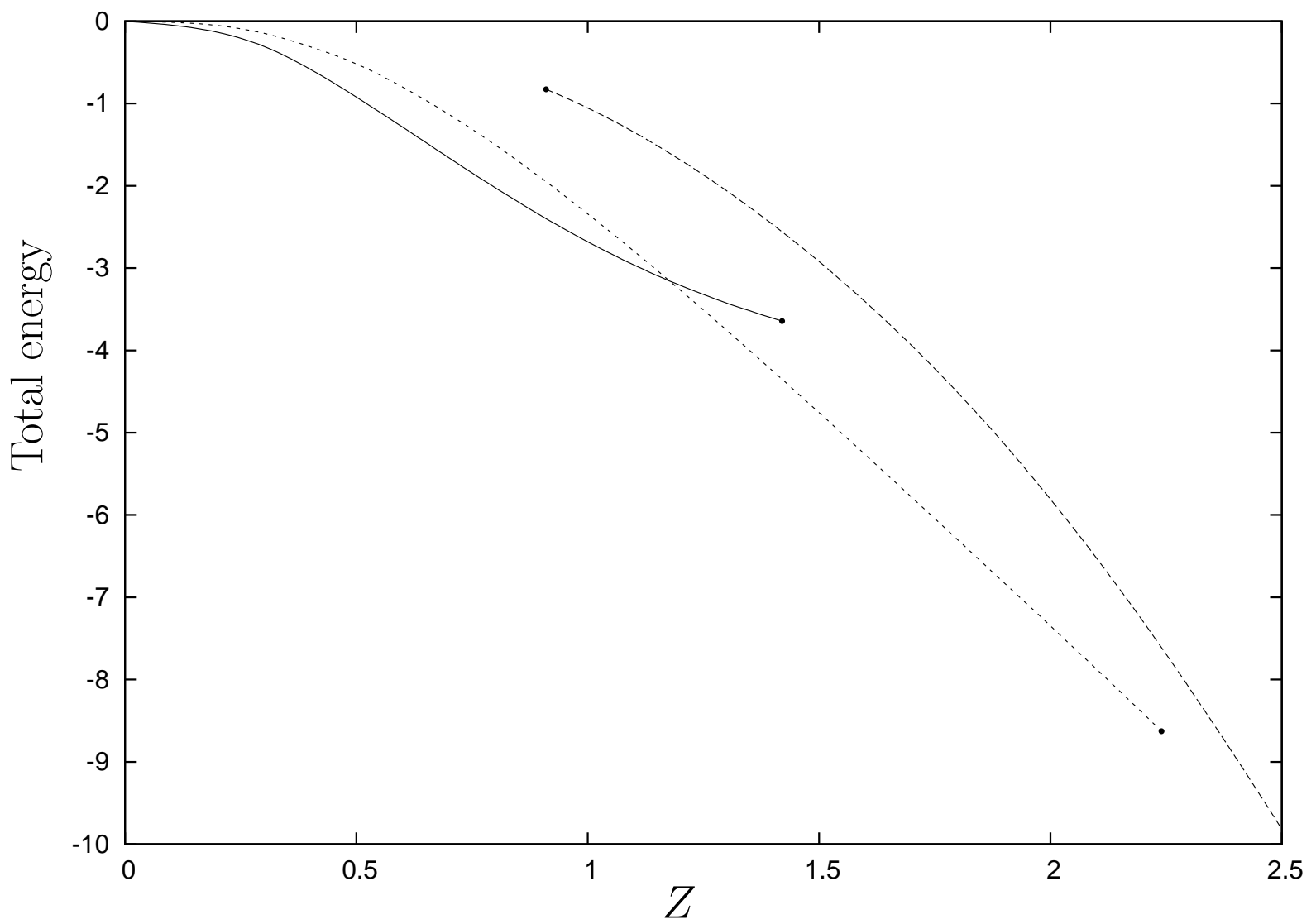

FIG. 7: Total energy $E_{T}$ in Ry vs $Z$ for two-electron systems in equilibrium at $R=R_{e q}:(2 Z, e, e)$ (solid line) and $(3 Z, e, e)$ (dashed line), and for comparison for $(Z, e, e)$ (long-dashed line). Both curves intersect at $Z=1.1767$. Solid line ends at $Z=Z_{c r}^{(1)}=2.250$. Dashed line ends at $Z=Z_{c r}^{(2)}=1.433$.

With such a function the expected relative accuracy in total energy is $\approx 10^{-3}$. The total energy $E\left(Z, R=R_{e q}\right)$ is presented at Fig. 7 and the equilibrium distance is at Fig. 8 , Both curves are smooth without any indication to a special feature at the physical charge 


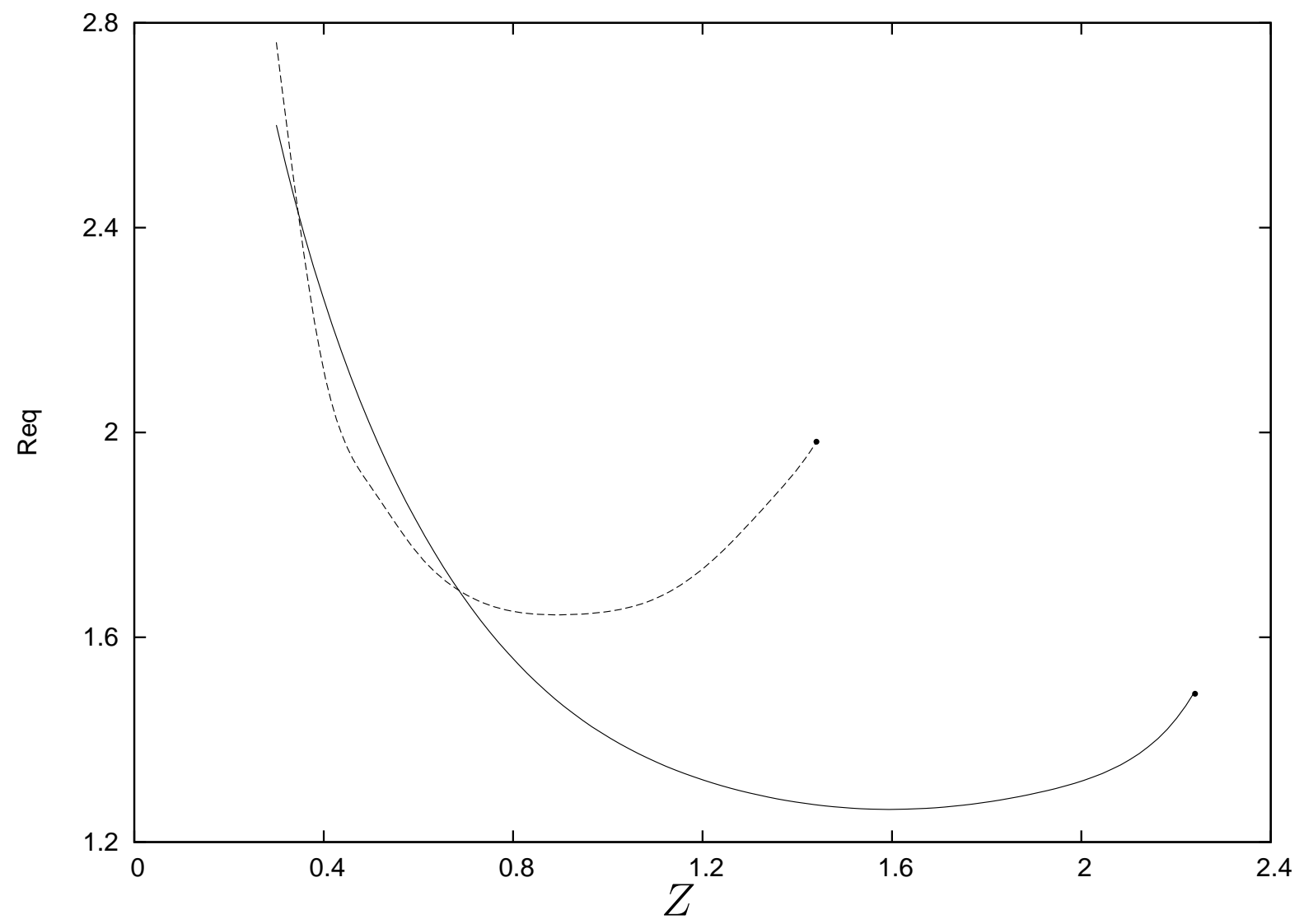

FIG. 8: Equilibrium distance $R_{e q}$ in a.u. of system $(2 Z, e, e)$ (solid line) and $(3 Z, e, e)$ (dashed line) as functions of the charge $Z$. Curves cross twice at $Z=0.3460$ and at $Z=0.6851$. Solid line ends at $Z=Z_{c r}^{(1)}=2.250$. Dashed line ends at $Z=Z_{c r}^{(2)}=1.433$.

$Z=1,2$. At charge $Z=1.1767$ the energy curves for $(2 Z, e, e)$ and $(3 Z, e, e)$ intersect. The equilibrium distance is a smooth curve which has a minimum $R_{e q}=1.264$ a.u. at $Z=1.596$ and it grows to infinity with a decrease of $Z$. At critical charge $Z=2.250$ the equilibrium distance, where the potential curve $E=E(R)$ has the saddle point, is equal to 1.532 a.u.

Behavior of the energy as function of the charge close to critical charge $Z<Z_{c r}$ is given by the terminated Puiseux expansion (see (7)):

$$
\begin{aligned}
E(Z) & =-8.6835+5.5238\left(Z_{c r}-Z\right)-0.2982\left(Z_{c r}-Z\right)^{3 / 2} \\
& -0.3166\left(Z_{c r}-Z\right)^{2}+0.3577\left(Z_{c r}-Z\right)^{5 / 2}+\ldots
\end{aligned}
$$

where the critical point is

$$
Z_{c r}=2.250
$$

The fit (31) is based on data from the domain $Z \in[1.80,2.22]$ (12 points, see Table IV). 
This behavior indicates that critical point might be a square-root branch point.

\begin{tabular}{|c|c|c|}
\hline$Z$ & $E_{T}$ & Fit \\
\hline \hline 0.30 & -0.1464 & -0.1496 \\
\hline 0.40 & -0.3091 & -0.3037 \\
\hline 0.50 & -0.5177 & -0.5211 \\
\hline 0.60 & -0.8001 & -0.7993 \\
\hline \hline 1.80 & -6.30334 & -6.30331 \\
\hline 1.90 & -6.82475 & -6.82475 \\
\hline 2.00 & -7.34768 & -7.34841 \\
\hline 2.10 & -7.87639 & -7.87623 \\
\hline 2.20 & -8.41058 & -8.41121 \\
\hline 2.22 & -8.52070 & -8.51953 \\
\hline
\end{tabular}

TABLE IV: Total energy $E_{T}$ of $(2 Z, e, e)$ in Ry at equilibrium distance vs $Z$ obtained using the trial function (30) compared to the result of the fit (31).

There are two points of crossing for the energy curve $(2 Z, e, e)$ displayed at Fig. 7. The first one is at $Z_{\text {cross }}^{(1)}=1.7026$ with $R_{e q}=1.268$ a.u. for the crossing of $(2 Z, e, e)$ and two atoms $(Z, e)$. The second one is $Z_{\text {cross }}^{(2)}=0.4501$ with $R_{e q}=2.126$ a.u. for the crossing of $(2 Z, e, e)$ and the $(2 Z, e)$ at $R_{e q}=2.119$ a.u. and two atoms $(Z, e)$.

For $Z \in(1.7026,2.250)$ the system $(2 Z, e, e)$ is metastable, there is decay channel

$$
(2 Z, e, e) \rightarrow(Z, e)+(Z, e)
$$

while for $Z \in(0.4501,1.7026)$ system is stable and for $Z<Z_{\text {cross }}^{(2)}=0.4501$ it seemingly gets metastable again with two decay channels

$$
\begin{gathered}
(2 Z, e, e) \rightarrow(2 Z, e)+e, \\
(2 Z, e, e) \rightarrow(Z, e)+(Z, e) .
\end{gathered}
$$

About the last domain we are not certain due to insufficient accuracy of our calculations.

A question to rise is a behavior of the total energy near the second critical point at $Z=0$ which is the singular point of the Schrödinger equation. Based on the fit of data from the 
domain $Z \in[0.1,0.6]$ (five points, see Table IV) we find that the Puiseux expansion becomes the Taylor expansion

$$
E_{T}=-0.6533 Z^{2}-4.1162 Z^{3}+2.5076 Z^{4}+\ldots .
$$

Such a behavior does not provide an indication to singular nature of the point $Z=0$. However, the total energy can not be analytically continued to $\operatorname{Re} Z<0$.

\section{B. $(3 Z, e, e)$}

The system $(3 Z, e, e)$ consists of three charged centers $Z$ and two electrons. For $Z=1$ it is celebrated $\mathrm{H}_{3}^{+}$molecular ion. It is obvious that for large $Z$ the system is unbound as well as for negative $Z$. Thus, there are two singular points: $Z=0$ where the potential "changes" sign and $Z_{c r}>2$ which is a critical point separating the domain of the existence from the domain of non-existence of the solutions in the Hilbert space. It seems natural that the ground state when exists is the spin-singlet state. Calculations (see below) show that $Z_{c r}=1.441$ at $R_{e q}=1.98$ a.u. The optimal geometrical configuration at equilibrium is the equilateral triangle. It was checked that this configuration is always stable with respect to small deviations. Thus, the system $(3 Z, e, e)$ exists for charge $0<Z<Z_{c r}$.

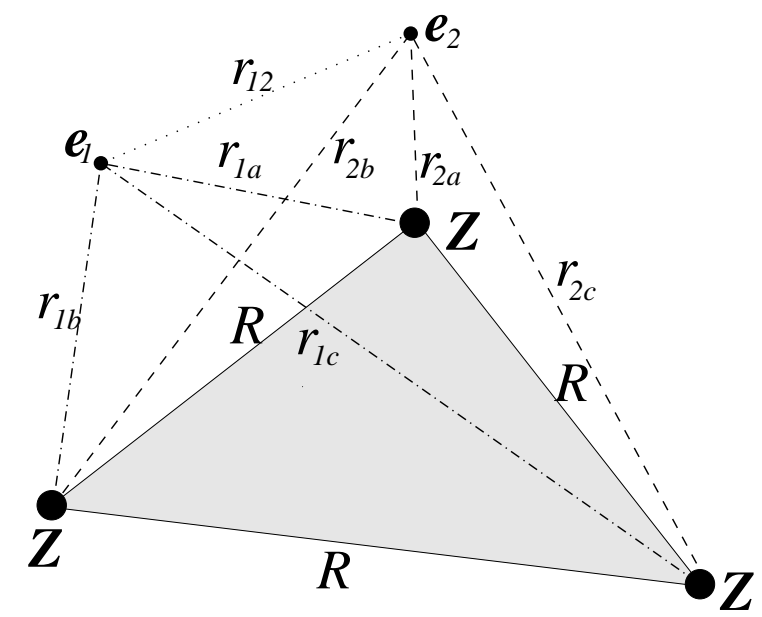

FIG. 9: Geometrical setting for $(3 Z, e, e)$ system

Trial Functions. The variational method was used to obtain all numerical results. In general, the basic trial function $\psi^{(j)}$ has the form of a symmetrized product of six $1 s$ Coulomb orbitals (Slater functions) and correlation function in exponential form (see [12] 
for a discussion),

$$
\begin{aligned}
\psi_{g} & =e^{-\alpha_{1} r_{1 a}-\alpha_{2} r_{1 b}-\alpha_{3} r_{1 c}-\alpha_{4} r_{2 a}-\alpha_{5} r_{2 b}-\alpha_{6} r_{2 c}+\gamma r_{12}} \\
& +e^{-\alpha_{1} r_{1 c}-\alpha_{2} r_{1 a}-\alpha_{3} r_{1 b}-\alpha_{4} r_{2 c}-\alpha_{5} r_{2 a}-\alpha_{6} r_{2 b}+\gamma r_{12}} \\
& +e^{-\alpha_{1} r_{1 b}-\alpha_{2} r_{1 c}-\alpha_{3} r_{1 a}-\alpha_{4} r_{2 b}-\alpha_{5} r_{2 c}-\alpha_{6} r_{2 a}+\gamma r_{12}} \\
& +e^{-\alpha_{1} r_{1 a}-\alpha_{2} r_{1 c}-\alpha_{3} r_{1 b}-\alpha_{4} r_{2 a}-\alpha_{5} r_{2 c}-\alpha_{6} r_{2 b}+\gamma r_{12}} \\
& +e^{-\alpha_{1} r_{1 c}-\alpha_{2} r_{1 b}-\alpha_{3} r_{1 a}-\alpha_{4} r_{2 c}-\alpha_{5} r_{2 b}-\alpha_{6} r_{2 a}+\gamma r_{12}} \\
& +e^{-\alpha_{1} r_{1 b}-\alpha_{2} r_{1 a}-\alpha_{3} r_{1 c}-\alpha_{4} r_{2 b}-\alpha_{5} r_{2 a}-\alpha_{6} r_{2 c}+\gamma r_{12}} \\
& +e^{-\alpha_{1} r_{2 a}-\alpha_{2} r_{2 b}-\alpha_{3} r_{2 c}-\alpha_{4} r_{1 a}-\alpha_{5} r_{1 b}-\alpha_{6} r_{1 c}+\gamma r_{12}} \\
& +e^{-\alpha_{1} r_{2 c}-\alpha_{2} r_{2 a}-\alpha_{3} r_{2 b}-\alpha_{4} r_{1 c}-\alpha_{5} r_{1 a}-\alpha_{6} r_{1 b}+\gamma r_{12}} \\
& +e^{-\alpha_{1} r_{2 b}-\alpha_{2} r_{2 c}-\alpha_{3} r_{2 a}-\alpha_{4} r_{1 b}-\alpha_{5} r_{1 c}-\alpha_{6} r_{1 a}+\gamma r_{12}} \\
& +e^{-\alpha_{1} r_{2 a}-\alpha_{2} r_{2 c}-\alpha_{3} r_{2 b}-\alpha_{4} r_{1 a}-\alpha_{5} r_{1 c}-\alpha_{6} r_{1 b}+\gamma r_{12}} \\
& +e^{-\alpha_{1} r_{2 c}-\alpha_{2} r_{2 b}-\alpha_{3} r_{2 a}-\alpha_{4} r_{1 c}-\alpha_{5} r_{1 b}-\alpha_{6} r_{1 a}+\gamma r_{12}} \\
& +e^{-\alpha_{1} r_{2 b}-\alpha_{2} r_{2 a}-\alpha_{3} r_{2 c}-\alpha_{4} r_{1 b}-\alpha_{5} r_{1 a}-\alpha_{6} r_{1 c}+\gamma r_{12}}
\end{aligned}
$$

It is worth noting that a linear superposition of three functions of a type (33) leads to the most accurate energy for the $\mathrm{H}_{3}^{+}$-molecule for lowest spin-triplet state in linear configuration ${ }^{3} \Sigma_{u}$ among a few parametric trial functions giving a relative accuracy $\sim 10^{-3}[13]$.

With such a function (33) the expected relative accuracy in total energy is $\approx 10^{-3}$. The total energy $E\left(Z, R=R_{e q}\right)$ of $(3 Z, e, e)$ is presented at Fig. 7 and the equilibrium distance is at Fig. 8. The optimal geometrical configuration at equilibrium is always the equilateral triangle. It was checked that this configuration is always stable with respect to small deviations. Both curves are smooth without any indication to a special feature at the physical charge $Z=1$. At charge $Z=1.1767$ the energy curves for $(2 Z, e, e)$ and $(3 Z, e, e)$ intersect. This crossing separates the domain of stability from metastability of the system $(3 Z, e, e)$ : at $Z>1.1767(3 Z, e, e)$ can decay to $(2 Z, e, e)+Z$. The equilibrium distance $R_{e q}$ is a smooth curve which has a minimum $R_{e q}=1.643$ a.u. at $Z=0.8981$, it grows to infinity with a decrease of $Z$. Two equilibrium distances curves for $(2 Z, e, e)$ and $(3 Z, e, e)$ intersect twice for $Z=0.6851$ with $R_{e q}=1.6917$ a.u. and for $Z=0.3460$ with $R_{e q}=2.4297$ a.u.

Behavior of the energy as function of the charge $Z$ close to critical charge $Z<Z_{c r}$ is 
given by the Puiseux expansion (see (7)):

$$
\begin{aligned}
E(Z) & =-3.6798+1.7613\left(Z_{c r}-Z\right)-0.5009\left(Z_{c r}-Z\right)^{3 / 2} \\
& +1.5164\left(Z_{c r}-Z\right)^{2}+0.6143\left(Z_{c r}-Z\right)^{5 / 2}+\ldots,
\end{aligned}
$$

where the critical point is

$$
Z_{c r}=1.441
$$

The fit (34) is based on data from the domain $Z \in[1.20,1.42]$ ( 7 points, see Table V). This behavior indicates that critical point seems to be a square-root branch point.

\begin{tabular}{|c|c|c|}
\hline$Z$ & $E_{T}$ & Fit \\
\hline 0.30 & -0.3051 & -0.3058 \\
\hline 0.40 & -0.5820 & -0.5809 \\
\hline 0.50 & -0.9216 & -0.9224 \\
\hline 0.60 & -1.2870 & -1.2868 \\
\hline \hline 1.20 & -3.2097 & -3.2095 \\
\hline 1.25 & -3.3197 & -3.3206 \\
\hline 1.30 & -3.4251 & -3.4237 \\
\hline 1.35 & -3.5185 & -3.5196 \\
\hline 1.40 & -3.6100 & -3.6094 \\
\hline 1.41 & -3.6269 & -3.6268 \\
\hline 1.42 & -3.6436 & -3.6440 \\
\hline
\end{tabular}

TABLE V: Total energy $E_{T}$ of $(3 Z, e, e)$ in Ry at equilibrium vs $Z$ obtained using (33) compared to the result of the fit (34).

There are three points of crossing for the energy curve $(3 Z, e, e)$ displayed at Fig. 7 , The first one is $Z_{\text {cross }}^{(1)}=1.3566$ with $R_{e q}=1.881$ a.u. for the crossing of $(2 Z, e)$ at $R_{e q}=2.406$ a.u. and the $(Z, e)$. The second one is $Z_{\text {cross }}^{(2)}=1.3137$ with $R_{e q}=1.837$ a.u. for the crossing with two atomic $(Z, e)$ systems. The third one is $Z_{\text {cross }}^{(3)}=1.1767$ with $R_{e q}=1.716$ a.u. for the crossing with the $(2 Z, e, e)$ at $R_{e q}=1.329$ a.u. 
For charges $Z \in(1.3566,1.4407)$ the system is metastable with three decay channels

$$
\begin{aligned}
& (3 Z, e, e) \rightarrow(2 Z, e)+(Z, e) \\
& (3 Z, e, e) \rightarrow 2(Z, e)+Z \\
& (3 Z, e, e) \rightarrow(2 Z, e, e)+Z
\end{aligned}
$$

For charges $Z \in(1.3137,1.3566)$ the system is metastable with two decay channels

$$
\begin{aligned}
& (3 Z, e, e) \rightarrow 2(Z, e)+Z \\
& (3 Z, e, e) \rightarrow(2 Z, e, e)+Z
\end{aligned}
$$

For charges $Z \in(1.1767,1.3137)$ the system is metastable with one decay channel

$$
(3 Z, e, e) \rightarrow(2 Z, e, e)+Z
$$

Eventually, for charges $Z \in(0.2989,1.1767)$ the system becomes stable. For charges $Z<$ 0.2989 the system can be either in the form $(3 Z, e)+e$ or $(2 Z, e)+(Z, e)$. The accuracy of our calculations do not allow us to make a definite statement.

Another question to rise is a behavior of the total energy near the second critical point at $Z_{c r}=0$ which is the singular point of the Schrödinger equation. Based on the fit of data from the domain $Z \in[0.1,0.5]$ (five points, see Table V) we find that the Puiseux expansion becomes the Taylor expansion

$$
E_{T}=-0.7198 Z^{2}-11.9676 Z^{3}+14.0751 Z^{4}+\ldots .
$$

Such a behavior does not provide an indication to singular nature of the point $Z=0$. However, the total energy can not be analytically continued to $\operatorname{Re} Z<0$.

\section{Conclusions}

In this paper we calculated for the first time the critical charges of five simple 1-2 electron molecular systems: $(2 Z, e),(3 Z, e),(4 Z, e),(2 Z, e, e),(3 Z, e, e)$ under the assumption that the $Z$-charges are static and found their equilibrium configurations. It was also found that for all those systems the total energy and equilibrium distance vs $Z$ are smooth curves without any indication to charge quantization. For all studied systems the optimal geometric configuration is the most symmetric being the equilateral triangle for $(3 Z, e),(3 Z, e, e)$ and 
tetrahedron for $(4 Z, e)$. It was checked that this configuration is always stable with respect to small deviations. It seems natural to assume that for $(4 Z, e, e)$ the tetrahedron as the optimal geometrical configuration would occur. It would be interesting to study the optimal geometrical configuration for five (or more) $Z$-center cases, in particular, for $(5 Z, e)$.

It is evident the existence of the critical charge for any one electron system $(n Z, e)$, since the potential has a form $V=-A Z+B Z^{2}$ with $A, B>0$ and always becomes negative if the charge $Z$ is small enough. The critical charge behaves like $Z_{c r, n} \propto n^{\alpha}$ with some $\alpha<0$ at large $n$. However, the question about stability of $(n Z, e)$ at $Z<Z_{c r, n}$ remains unclear to the present authors. Probably, a similar conclusion can be made for two electron systems.

\section{Acknowledgments}

The authors are grateful to J. C. López Vieyra for helpful discussions, important assistance with computer calculations and for their interest in the present work. This work was supported in part by the university program FENOMEC and by the PAPIIT grant IN115709 and CONACyT grant 58962-F (Mexico). H.M.C. thanks to PhD study support program through CONACyT grant 58962-F.

[1] J.D. Baker, D.E. Freund, R.N. Hill, and J.D. Morgan III, Radius of convergence and analytic behavior of the $1 / Z$ expansion, Phys. Rev. A41 (1990) 1247

[2] J. Zamastil, J. Cizek, L. Skala, and M. Simanek, Convergence study of the $1 / Z$ expansion for the energy levels of two-electron atoms, Phys. Rev. A81 (2010) 032118

[3] Q. Shi and S. Kais, Mol. Phys. 98, 1485 (2000)

[4] A.V. Turbiner and J.C. Lopez Vieyra, One-electron Molecular Systems in a Strong Magnetic Field, Phys.Repts. 424, 309-396 (2006)

[5] L.D. Landau and E.M. Lifshitz, Quantum Mechanics, Non-relativistic Theory (Course of Theoretical Physics vol 3), 3rd edn (Oxford:Pergamon Press), 1977 
[6] A.V. Turbiner and H. Olivares-Pilon, Hydrogen molecular ion $\mathrm{H}_{2}^{+}$: a solution (in preparation)

[7] T.K. Rebane, Sov.Phys. ZhETF, 71, 1055 (1995)

[8] H. Medel, A. Alijah and A.V. Turbiner, About non-existence of the molecular ion $\mathbf{H}_{\mathbf{3}}^{++}$, (invited contribution on the occasion of 80th birthday of R. Zahradnik) COLLECTION of Czechoslovak Chemical Communications 73, 1271-1280 (2008)

[9] A.V. Turbiner, Usp. Fiz. Nauk. 144, 35 (1984) Sov.Phys. - Uspekhi 27, 668 (1984) (English Translation)

[10] A.V. Turbiner, Yad. Fiz. 46, 204 (1987) Sov. Journ. of Nucl. Phys. 46, 125 (1987) (English Translation)

[11] A.V. Turbiner and J.C. Lopez Vieyra, $\mathrm{H}_{3}^{++}$in a strong magnetic field: triangular configuration, Phys.Rev. A66, 023409 (2002)

[12] A.V. Turbiner and N.L. Guevara, A note about the ground state eigenfunction of the hydrogen molecule, COLLECTION of Czechoslovak Chemical Communications 72, 164-170 (2007)

[13] A.V. Turbiner, N.L. Guevara and J.C. Lopez Vieyra, $H_{3}^{+}$molecular ion in a magnetic field: Linear parallel configuration Phys.Rev. A75, 053408 (2007)

[14] The Hamiltonian (11) at $k=1$ allows the separation of variables in elliptic coordinates (see e.g. [5]). Every function $\psi_{1,2,3}$ admits a factorization in elliptic coordinates, although their linear superposition (6), in general, does not. Factorization implies imposing a constraint on parameters $\alpha$ 's. We do not impose such a constraint, however, for the parameters obtained as a result of the variational optimization the constraint is almost fulfilled. It indicates to a quality of the trial function (6). 